\title{
Laparoscopic Roux-en-Y gastric bypass for excess weight and diabetes: a multicenter retrospective cohort study in China
}

\author{
Wah Yang ${ }^{1}$, Shaihong Zhu ${ }^{2}$, Zhong Cheng ${ }^{3}$, Nengwei Zhang ${ }^{4}$, Liangping Wu ${ }^{5}$, Yi Chen ${ }^{3}$, Jingge Yang ${ }^{1}$, \\ Shuqing Yu ${ }^{1}$, Tengfei Yang ${ }^{6}$, Ding Ding ${ }^{6}$, Jason R. Waggoner ${ }^{7}$, Michael L. Schwiers ${ }^{7}$, Elliott J. Fegelman ${ }^{7}$, \\ Cunchuan Wang ${ }^{1}$
}

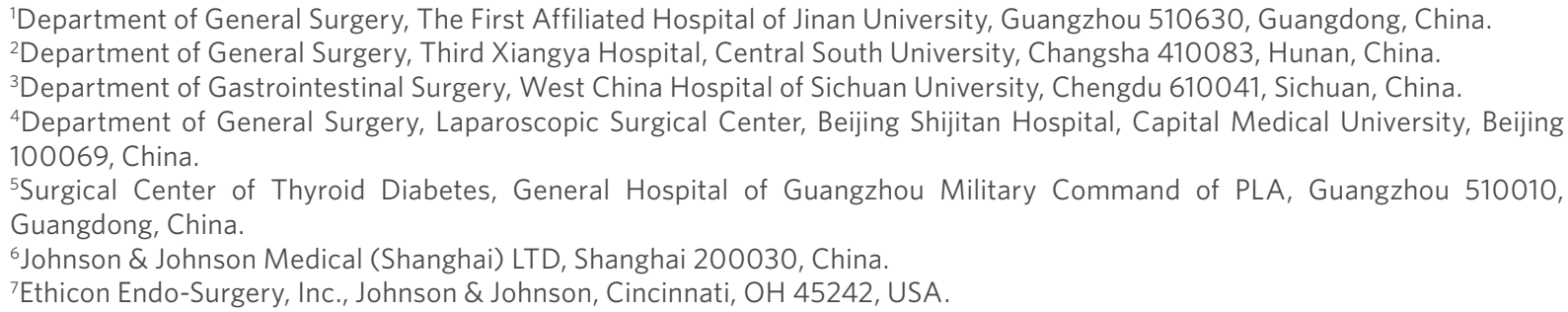

Correspondence to: Prof. Cunchuan Wang, Department of General Surgery, First Affiliated Hospital of Jinan University, 613 Huangpu Avenue West, Guangzhou 510630, Guangdong, China. E-mail: twcc@jnu.edu.cn; Prof. Shaihong Zhu, Department of General Surgery, Third Xiangya Hospital, Central South University, No. 138 Tongzipo Road, Yuelu District Changsha, Hunan 410013, China. E-mail: shzhu@mail.csu.edu.cn; Prof. Nengwei Zhang, Department of General Surgery, Laparoscopic Surgical Center, Beijing Shijitan Hospital, Capital Medical University, No.10 Tieyilu, Haidian District, Beijing 100069, China. E-mail: zhangnw1@sohu.com; Prof. Zhong Cheng, Department of Gastrointestinal Surgery, West China Hospital of Sichuan University, No.37 Guoxue Alley, Wuhou District, Chengdu 610041, Sichuan, China. E-mail: zhongcheng63@126.com; Prof. Liangping Wu, Surgical Center of Thyroid Diabetes, General Hospital of Guangzhou Military Command of PLA, No. 111 Liuhualu, Guangzhou 510010, Guangdong, China. E-mail:drwulp@163.com

How to cite this article: Yang W, Zhu S, Cheng Z, Zhang N, Wu L, Chen Y, Yang J, Yu S, Yang T, Ding D, Waggoner JR, Schwiers ML, Fegelman EJ, Wang C. Laparoscopic Roux-en-Y gastric bypass for excess weight and diabetes: a multicenter retrospective cohort study in China. Mini-invasive Surg 2021;5:11. http://dx.doi.org/10.20517/2574-1225.2021.06

Received: 15 Jan 2021 First Decision: 5 Feb 2021 Revised: 18 Feb 2021 Accepted: 24 Feb 2021 Published: 9 Mar 2021

Academic Editor: Giulio Belli Copy Editor: Yue-Yue Zhang Production Editor: Yue-Yue Zhang

\begin{abstract}
Aim: The aims of this study were to better understand the outcomes of Roux-en-Y gastric bypass (RYGB) surgery in patients across multiple hospitals in China along with patients with type 2 diabetes mellitus (T2DM) and to explore the potential preoperative predictors of diabetes outcomes after RYGB.
\end{abstract}

Methods: This was a retrospective cohort study in Chinese patients who underwent laparoscopic RYGB at five Chinese hospitals from April 2009 to December 2014 and returned for follow-up approximately one-year post-

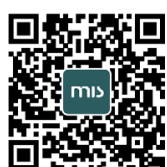


surgery. The STROCSS guideline checklist was applied.

Results: In total, 130 patients underwent RYGB: 85 males and 45 females; age, $43.4 \pm 11.3$ years; and preoperative body mass index (BMI), $33.1 \pm 9.0 \mathrm{~kg} / \mathrm{m}^{2}$. Of those, $103(79.2 \%)$ had T2DM duration of $6.6 \pm 4.7$ years and preRYGB HbA1c of $8.1 \pm 1.9 \%$. Among the patients with T2DM, glycemic control (HbA1c $<7.0 \%$ ) increased from $28.7 \%$ before surgery to $79.3 \%$ at 12 months post-procedure, with a concurrent reduction in the use of antihyperglycemic agents, including a reduction in insulin requirement from $55.4 \%$ to $27.0 \%$. The percentage of excess weight loss was $-42.8 \pm 44.2 \%$. Among 71 patients with T2DM and data about remission status, 14 (19.7\%) achieved T2DM remission at 12 months post-surgery. Age and duration of T2DM were lower in the remission group, while baseline $\mathrm{BMI}$ and weight were higher compared with the non-remission group.

Conclusion: RYGB may be effective for weight loss and T2DM control in Chinese patients, and outcomes are consistent with the literature in Western populations. Younger patients with T2DM and with a higher BMI presurgery and shorter duration of T2DM were more likely to achieve T2DM remission.

Keywords: Type 2 diabetes, obesity, roux-en-Y gastric bypass, glycemic control, remission

\section{INTRODUCTION}

World Health Organization (WHO) estimates that 422 million adults globally were living with type 2 diabetes mellitus (T2DM) in 2014 and that the prevalence of T2DM has doubled since $1980^{[1]}$. China has almost 115 million patients with T2DM, with an adult diabetes prevalence of $9.8 \%$ that is rapidly increasing, presenting in individuals with higher insulin resistance but with lower body mass index (BMI) and approximately 10 years younger than their Western counterparts ${ }^{[2-4]}$. A review of the literature about bariatric surgery in China showed a significant increase in the number of procedures performed in China between 2001 and 2015 (a total of 7779 procedures in this period, from 47 surgeries during 2001-2005 to 795 during 2006-2010 and 6937 during 2011-2015); in addition, the proportion of procedures performed to treat obesity-related comorbidities (defined as metabolic surgery) increased from $0 \%$ of the total number of procedures performed in 2001 to $70 \%$ by $2015^{[5]}$.

While the growing obesity pandemic is considered a major factor in the growth of T2DM prevalence ${ }^{[6]}$, central adiposity, not BMI per se, is considered a primary factor in the rise of T2DM in China ${ }^{[7]}$ and other regions of $\mathrm{Asia}^{[8]}$. BMI distributions in the adult populations differ between the United States and China. Approximately $31 \%$ of adults in China ${ }^{[9]}$ are classified as overweight (BMI $\geq 24$ to $<28.0 \mathrm{~kg} / \mathrm{m}^{2}$ ) and $12 \%$ as obese $\left(\mathrm{BMI} \geq 28 \mathrm{~kg} / \mathrm{m}^{2}\right)$ compared to $40 \%$ obese (BMI $\left.\geq 30.0 \mathrm{~kg} / \mathrm{m}^{2}\right)$ and $8 \%$ severely obese (BMI $\geq 40.0$ $\left.\mathrm{kg} / \mathrm{m}^{2}\right)$ in the United States in $2016^{[10]}$. Therefore, the WHO has defined obesity in terms of abdominal obesity, a waist-hip ratio above 0.90 for males and 0.85 for females, or a BMI $>30.0 \mathrm{~kg} / \mathrm{m}^{2[6,11]}$ and has recommended health action (such as bariatric surgery) in Asians with T2DM at a BMI $2.5 \mathrm{~kg} / \mathrm{m}^{2}$ lower than in other ethnicities (i.e, BMI $27.5 \mathrm{~kg} / \mathrm{m}^{2} v s .30 .0 \mathrm{~kg} / \mathrm{m}^{2}$ ). Globally, bariatric metabolic surgeries such as Roux-en-Y gastric bypass (RYGB) and sleeve gastrectomy have emerged as the most effective interventions for sustained weight and diabetes control in patients who are obese ${ }^{[11]}$. Given the burden of disease in China, metabolic surgery is being undertaken in some patients at even lower BMI ${ }^{[12,13]}$. Studies showed that laparoscopic RYGB could be beneficial in patients with BMI $<28 \mathrm{~kg} / \mathrm{m}^{2}$, or even $<27.5 \mathrm{~kg} / \mathrm{m}^{2[12,13]}$.

Although the surgical techniques have been described extensively, evidence of laparoscopic RYGB in Chinese patients who are overweight or obese, with or without T2DM, is still limited. This multicenter study aimed to examine the health outcomes after RYGB surgery and determine the potential preoperative predictors of diabetes remission after RYGB surgery. 


\section{METHODS}

\section{Study design}

This was a retrospective cohort study in Chinese patients who underwent an RYGB procedure between April 2009 and December 2014 at five Chinese academic urban hospitals and returned for follow-up approximately one-year post-surgery. This study was approved by each site's ethics committee, including a waiver for informed consent due to the retrospective nature of this study. The study was registered at ChiCTR.org.cn (\#ChiCTR-OOC-15006387). The study was reported in accordance with the STROCSS guideline checklist ${ }^{[14]}$.

The inclusion criteria were: (1) underwent an RYGB procedure; (2) aged 20-60 years; and (3) had outcome data recorded [at least one of glycated hemoglobin (HbA1c), fasting plasma glucose, or fasting insulin levels] in their medical charts at approximately 12 months after surgery.

\section{Study interventions}

All participating hospitals assessed each patient who underwent RYGB through a multidisciplinary and integrated health unit, including a bariatric surgeon, endocrinologist, psychiatrist, cardiologist, and dietician. Weight, BMI, T2DM duration, anthropometric measures, systolic and diastolic blood pressures, glycemic control (HbA1c, fasting blood glucose, and insulin), lipid profile, and other laboratory and clinical evaluations recorded in the patient's medical record were analyzed. Given the retrospective design, not all outcome measures were available for all patients, and those outcomes available were not always available at all study time points.

\section{Outcomes}

The primary outcome was the resolution of T2DM. The secondary outcomes were weight reduction, improvements in glycemic control, vital signs, blood lipids, liver function, and adverse events (AEs).

\section{Statistical analysis}

The study was not statistically powered, and data from all patients who had an RYGB procedure during the study period and met the eligibility criteria were analyzed. For the total study population, interest focused on changes in anthropometric characteristics, vital signs, glycemic parameters, serum lipids, and liver function tests. In addition, for subjects with T2DM, changes in concomitant T2DM medication were of interest as well as the remission of T2DM, which was defined as fasting glucose levels $<110 \mathrm{mg} / \mathrm{dL}$ and $\mathrm{HbA} 1 \mathrm{c}<6.0 \%$ without the use of anti-hyperglycemic agents (AHAs) at 12 months after surgery.

Summary statistics for the outcome parameters were calculated, as well as their change from baseline. For all analyses, baseline was defined as the last available measurement taken on or before the date of RYGB surgery. For the mean change from baseline, $95 \%$ confidence intervals were estimated, and the one-sample $t$-test or the Wilcoxon rank-sum test was applied. No multiplicity adjustments were made to $P$-values for testing the change from baseline. Given the retrospective design of the study and sparseness of data at all available time points post-surgery for some parameters, the last observation carried forward (LOCF) approach was used. For each parameter, the latest value observed in the first 12 months after surgery was identified and used to evaluate the change from baseline to Month 12. Change in BMI was summarized by baseline BMI subgroup based on the WHO cutoff points. A significance level of 0.05 was considered statistically significant, and all reported p-values are nominal $P$-values.

To explore which factors could be associated with T2DM remission or non-remission, univariable and multivariable analyses were performed. Summary statistics for baseline demographic and clinical characteristics as well as post-surgery weight and BMI change were generated for patients with and without 
Table 1. Demographics and baseline characteristics

\begin{tabular}{|c|c|c|c|c|}
\hline \multirow{2}{*}{ Characteristic } & \multicolumn{2}{|c|}{ Overall $(n=130)$} & \multicolumn{2}{|r|}{ T2DM $(n=103)$} \\
\hline & $n$ & Mean \pm SD/n (\%) & $n$ & Mean \pm SD/n (\%) \\
\hline Age (years) & 130 & $43.4 \pm 11.3$ & 103 & $46.2 \pm 10.1$ \\
\hline \multicolumn{5}{|l|}{ Sex } \\
\hline Female & 130 & $45(34.6)$ & 103 & $33(32.0)$ \\
\hline Male & 130 & $85(65.4)$ & 103 & $70(68.0)$ \\
\hline $\mathrm{BMI}\left(\mathrm{kg} / \mathrm{m}^{2}\right)$ & 127 & $33.1 \pm 9.0$ & 101 & $31.2 \pm 7.9$ \\
\hline Weight $(\mathrm{kg})$ & 130 & $94.7 \pm 29.6$ & 103 & $87.9 \pm 24.2$ \\
\hline Waist circumference $(\mathrm{cm})$ & 102 & $108.0 \pm 21.4$ & 86 & $104.2 \pm 18.7$ \\
\hline Female & 35 & $108.2 \pm 18.4$ & 27 & $105.5 \pm 19.3$ \\
\hline Male & 67 & $107.9 \pm 22.9$ & 59 & $103.6 \pm 18.6$ \\
\hline Waist-to-hip ratio & 76 & $0.96 \pm 0.10$ & 75 & $0.96 \pm 0.10$ \\
\hline Female & 24 & $0.93 \pm 0.14$ & 24 & $0.93 \pm 0.14$ \\
\hline Male & 52 & $0.97 \pm 0.06$ & 51 & $0.97 \pm 0.06$ \\
\hline Duration of T2DM (years) & NA & NA & 102 & $6.6 \pm 4.7$ \\
\hline
\end{tabular}

BMI: Body mass index; NA: not applicable; SD: standard deviation; T2DM: type 2 diabetes mellitus.

T2DM remission. Logistic regression analyses with T2DM remission as the dependent variable were also performed using backward selection to determine what variables were independently associated with T2DM remission when considering all predictors simultaneously. All statistical analyses were performed with SAS ${ }^{\circledast}$, Cary, NC.

\section{RESULTS}

\section{Patient characteristics}

In total, 130 Han Chinese patients met the eligibility criteria, of whom 103 patients (79.2\%) had a diagnosis of T2DM. Demographics and baseline characteristics are presented in Table 1.

\section{Surgical interventions and outcomes}

RYGB procedures and postoperative care were performed per the standard of care at each hospital. The mean length of the biliopancreatic limb was $74.9 \pm 37.0 \mathrm{~cm}$, and the Roux limb was $97.5 \pm 36.6 \mathrm{~cm}$. All 130 procedures were successfully completed laparoscopically across a broad BMI range of $20.8-65.3 \mathrm{~kg} / \mathrm{m}^{2}(2.4 \%$ for BMI 18.5 to $<23.0 \mathrm{~kg} / \mathrm{m}^{2}$; $29.9 \%$ for BMI 23.0 to $<27.5 \mathrm{~kg} / \mathrm{m}^{2} ; 27.6 \%$ for BMI 27.5 to $<32.5 \mathrm{~kg} / \mathrm{m}^{2}$; and $40.2 \%$ for BMI $>32.5 \mathrm{~kg} / \mathrm{m}^{2}$ ). The mean operative time was $179 \pm 59 \mathrm{~min}$. The mean length of stay (surgeryto-discharge) was $8.8 \pm 5.7$ nights.

For the total population, weight pre-surgery and at 12 months was available for 90 patients and was reduced by $16.5 \pm 12.8 \%$. Meaningful reductions in BMI were also observed $\left(-6.2 \pm 5.6 \mathrm{~kg} / \mathrm{m}^{2}\right)$ at $12 \mathrm{months}$ with LOCF. Excessive weight loss was not observed as the lowest postoperative BMI reported was $18.1 \mathrm{~kg} / \mathrm{m}^{2}$. Meaningful improvements were also observed in the total population through 12 months for glycemic control, vital signs, blood lipids, and liver function [Table 2]. Among 53 procedure-related AEs, 24 (45.3\%) were recorded as Clavien-Dindo Grade 1, 20 (37.7\%) were Grade 2, and 9 (17.0\%) were Grade 3. The more serious events (all Grade 3, no Grade 4) included ileus $(n=2)$, anastomotic leak $(n=1)$, anastomotic stenosis $(n=1)$, gastric fistula $(n=1)$, gastric ulcer $(n=1)$, intestinal obstruction $(n=1)$, post-procedural edema $(n=1)$, and small intestinal obstruction $(n=1)$. Six patients $(4.6 \%)$ reported nine AEs within 30 days after the procedure, including five patients (six AEs) with GI disorders. Five patients experienced AEs requiring reoperation, and these AEs included small bowel obstruction, anastomotic leakage, anastomotic stenosis, ileus, gastric fistula, and anastomotic edema. Every AE requiring reoperation was resolved.

\section{PATIENTS WITH T2DM AND RISK ANALYSIS}

Following RYGB surgery in patients with T2DM, statistically significant and clinically meaningful improvements in anthropometric characteristics and laboratory values were observed 12 months after 
Table 2. Anthropometric characteristics, vital signs, and laboratory values for patients with T2DM using last observation in Year 1 carried forward

\begin{tabular}{|c|c|c|c|c|c|}
\hline Variable & $n$ & Baseline & Month 12 & $\Delta, 0$ to $12 \mathrm{mo}$ & $\boldsymbol{P}$ \\
\hline \multicolumn{6}{|l|}{ Weight } \\
\hline Weight (kg) & 87 & $87.7 \pm 23.9$ & $72.7 \pm 20.4$ & $-15.0 \pm 15.2$ & $<0.001$ \\
\hline Change in weight (\%) & 87 & NA & NA & $-15.9 \pm 12.5$ & $<0.001$ \\
\hline$\% E W L^{\dagger}$ & 87 & NA & NA & $-42.8 \pm 44.2$ & $<0.001$ \\
\hline BMI $\left(\mathrm{kg} / \mathrm{m}^{2}\right)$ & 78 & $30.9 \pm 7.9$ & $24.9 \pm 5.8$ & $-6.0 \pm 5.5$ & $<0.001$ \\
\hline \multicolumn{6}{|l|}{ Blood pressure } \\
\hline Systolic blood pressure (mmHg) & 88 & $129.4 \pm 13.8$ & $123.0 \pm 14.3$ & $-6.4 \pm 15.9$ & $<0.001$ \\
\hline Diastolic blood pressure $(\mathrm{mmHg})$ & 88 & $80.3 \pm 9.2$ & $77.6 \pm 9.4$ & $-2.7 \pm 11.6$ & 0.034 \\
\hline \multicolumn{6}{|l|}{ Glycemic outcomes } \\
\hline $\operatorname{HbA1c}(\%)$ & 87 & $8.0 \pm 1.9$ & $6.1 \pm 1.5$ & $-1.9 \pm 2.2$ & $<0.001$ \\
\hline $\mathrm{FBG}(\mathrm{mg} / \mathrm{dL})$ & 100 & $165.8 \pm 64.9$ & $116.6 \pm 37.6$ & $-49.2 \pm 70.3$ & $<0.001$ \\
\hline Fasting C-peptide (ng/mL) & 85 & $2.5 \pm 1.5$ & $1.5 \pm 0.7$ & $-1.0 \pm 1.4$ & $<0.001$ \\
\hline Fasting insulin (miu/L) & 75 & $19.4 \pm 14.9$ & $7.7 \pm 8.8$ & $-11.7 \pm 15.0$ & $<0.001$ \\
\hline \multicolumn{6}{|l|}{ Serum lipids } \\
\hline $\mathrm{HDL}-\mathrm{C}(\mathrm{mg} / \mathrm{dL})$ & 95 & $44.6 \pm 12.1$ & $51.0 \pm 14.7$ & $6.4 \pm 114.0$ & $<0.001$ \\
\hline LDL-C (mg/dL) & 95 & $107.1 \pm 35.7$ & $84.2 \pm 23.7$ & $-22.9 \pm 33.9$ & $<0.001$ \\
\hline Triglycerides (mg/dL) & 94 & $229.9 \pm 254.4$ & $120.7 \pm 155.7$ & $-109.2 \pm 254.1$ & $<0.001$ \\
\hline $\mathrm{TC}(\mathrm{mg} / \mathrm{dL})$ & 95 & $188.0 \pm 54.9$ & $153.3 \pm 32.7$ & $-34.7 \pm 58.5$ & $<0.001$ \\
\hline \multicolumn{6}{|l|}{ Liver function } \\
\hline $\operatorname{ALT}(U / L)$ & 96 & $37.9 \pm 25.0$ & $27.4 \pm 15.9$ & $-10.5 \pm 27.8$ & $<0.001$ \\
\hline AST (U/L) & 88 & $30.1 \pm 16.5$ & $25.1 \pm 14.1$ & $-5.0 \pm 21.6$ & 0.032 \\
\hline
\end{tabular}

Data are shown as mean \pm standard deviation. ${ }^{\circ} \% \mathrm{EWL}$ is based on a target BMI of $19.0 \mathrm{~kg} / \mathrm{m}^{2}$. ALT: Alanine aminotransferase; AST: aspartate aminotransferase; BMI: body mass index; FBG: fasting blood glucose; HbA1c: glycosylated hemoglobin; HDL-C: high-density lipoprotein cholesterol; \%EWL: percent excess weight loss; LDL-C: low-density lipoprotein cholesterol; NA: not applicable; TC: total cholesterol.

surgery [Table 2]. There were significant reductions in the glycemic outcomes (HbA1c, fasting blood glucose, fasting c-peptide, and fasting insulin) from baseline to Month 12. In addition, patients had improved blood pressure values (systolic and diastolic), lipid values (increased high-density lipoprotein cholesterol and decreased low-density lipoprotein cholesterol, triglycerides, and total cholesterol), and liver function values (decreased alanine aminotransferase and aspartate aminotransferase). Patients lost $15.9 \pm 12.5 \%$ of their weight, with higher weight loss observed in those with higher BMI at baseline [Tables 2 and 3]. Severely obese individuals $\left(B M I \geq 32.5 \mathrm{~kg} / \mathrm{m}^{2}\right)$ lost $20.7 \pm 16.5 \%$ of their weight on average. Figure 1 demonstrates an overall trend towards reduced health risk based on BMI classification. The only patient with $\mathrm{BMI}<23 \mathrm{~kg} / \mathrm{m}^{2}$ remained in this category at 12 months. Among the 21 patients with BMI $23-27.5 \mathrm{~kg} / \mathrm{m}^{2}$ before surgery, $10(47.6 \%)$ remained in the same BMI category, while $11(52.4 \%)$ were downgraded to $<23$ $\mathrm{kg} / \mathrm{m}^{2}$. Among the 32 patients with BMI $>27.5 \mathrm{~kg} / \mathrm{m}^{2}$ before surgery, $9(28.1 \%)$ remained in the same BMI category, $13(40.6 \%)$ were downgraded to $23-27.5 \mathrm{~kg} / \mathrm{m}^{2}$, and $10(31.3 \%)$ were downgraded to $<23 \mathrm{~kg} / \mathrm{m}^{2}$. Therefore, $63 \%$ of the patients with T2DM reduced their WHO BMI risk category by at least one category after RYGB.

As shown in Figure 2, the percentage of patients diagnosed with T2DM and achieving glycemic control (HbA1c $<6.0 \%)$ significantly increased from baseline $(11.5 \%)$ to 12 months post-procedure $(56.3 \%)$. An overall reduction in the use of AHAs occurred during the first year after surgery [Figure 3], including a decrease in the number of patients with insulin requirement, from $55.4 \%$ at baseline to $27.0 \%$ over 12 months. Patients with T2DM requiring no AHA increased from $16.2 \%$ at baseline to $33.8 \%$ at 12 months post-procedure. The percentage of patients taking antihypertensive medication decreased (baseline to Month 12) from $28.4 \%$ to $18.9 \%$, and those taking dyslipidemia medication decreased from $8.1 \%$ to $4.1 \%$.

There were 71 patients with data available for the assessment of $\mathrm{T} 2 \mathrm{DM}$ remission and any potential predictive factor. Fifty-seven patients (80.3\%) showed improvements and near-remission and 14 patients 
Table 3. Anthropometric characteristics and laboratory values for patients with T2DM (stratified by BMI group) using last observation in Year 1 carried forward

\begin{tabular}{|c|c|c|c|c|c|c|c|c|}
\hline \multirow{3}{*}{ Variable } & \multicolumn{8}{|c|}{$\Delta, 0$ to $12 \mathrm{mo}$} \\
\hline & \multicolumn{2}{|c|}{$\begin{array}{l}\text { BMI Group 0 } \\
\left(<23.0 \mathrm{~kg} / \mathrm{m}^{2}\right)\end{array}$} & \multicolumn{2}{|c|}{$\begin{array}{c}\text { BMI Group I } \\
\left(23.0 \text { to }<27.5 \mathrm{~kg} / \mathrm{m}^{2}\right)\end{array}$} & \multicolumn{2}{|c|}{$\begin{array}{c}\text { BMI Group II } \\
\left(27.5 \text { to }<32.5 \mathrm{~kg} / \mathrm{m}^{2}\right)\end{array}$} & \multicolumn{2}{|c|}{$\begin{array}{l}\text { BMI Group III } \\
\left(\geq 32.5 \mathrm{~kg} / \mathrm{m}^{2}\right)\end{array}$} \\
\hline & $\bar{n}$ & Mean \pm SD & $n$ & Mean \pm SD & $n$ & Mean \pm SD & $n$ & Mean \pm SD \\
\hline \multicolumn{9}{|l|}{ Weight } \\
\hline Weight (\%) & 1 & -13.4 & 34 & $-11.8 \pm 10.2$ & 28 & $-16.9 \pm 9.8$ & 24 & $-20.7 \pm 16.5$ \\
\hline $\mathrm{BMI}\left(\mathrm{kg} / \mathrm{m}^{2} \%\right)$ & 1 & -8.2 & 31 & $-12.7 \pm 9.4$ & 28 & $-18.0 \pm 9.4$ & 18 & $-27.1 \pm 14.4$ \\
\hline \multicolumn{9}{|l|}{ Glycemic outcomes } \\
\hline $\operatorname{HbA1c}(\Delta \%)$ & 1 & -1.7 & 34 & $-1.6 \pm 2.2$ & 27 & $-1.9 \pm 2.3$ & 24 & $-2.5 \pm 2.1$ \\
\hline $\mathrm{FBG}(\mathrm{mg} / \mathrm{dL})$ & 3 & $-16.9 \pm 19.8$ & 35 & $-51.6 \pm 70.0$ & 31 & $-36.2 \pm 78.1$ & 30 & $-66.8 \pm 61.1$ \\
\hline \multicolumn{9}{|l|}{ Serum lipids } \\
\hline $\mathrm{HDL}-\mathrm{C}(\mathrm{mg} / \mathrm{dL})$ & 3 & $-8.8 \pm 4.8$ & 32 & $6.6 \pm 15.2$ & 31 & $6.5 \pm 15.5$ & 28 & $7.1 \pm 10.5$ \\
\hline LDL-C (mg/dL) & 3 & $-25.6 \pm 14.4$ & 32 & $-15.5 \pm 40.6$ & 31 & $-21.6 \pm 31.4$ & 28 & $-32.7 \pm 29.0$ \\
\hline Triglycerides (mg/dL) & 3 & $-31.0 \pm 12.9$ & 32 & $-158.5 \pm 393.1$ & 30 & $-53.5 \pm 152.9$ & 28 & $-123.3 \pm 112.2$ \\
\hline $\mathrm{TC}(\mathrm{mg} / \mathrm{dL})$ & 3 & $-36.3 \pm 28.2$ & 32 & $-26.4 \pm 59.1$ & 31 & $-37.7 \pm 76.9$ & 28 & $-41.5 \pm 33.7$ \\
\hline
\end{tabular}

BMI: Body mass index; FBG: fasting blood glucose; HbA1c: glycosylated hemoglobin; HDL-C: high-density lipoprotein cholesterol; LDL-C: low-density lipoprotein cholesterol; SD: standard deviation; TC: total cholesterol.

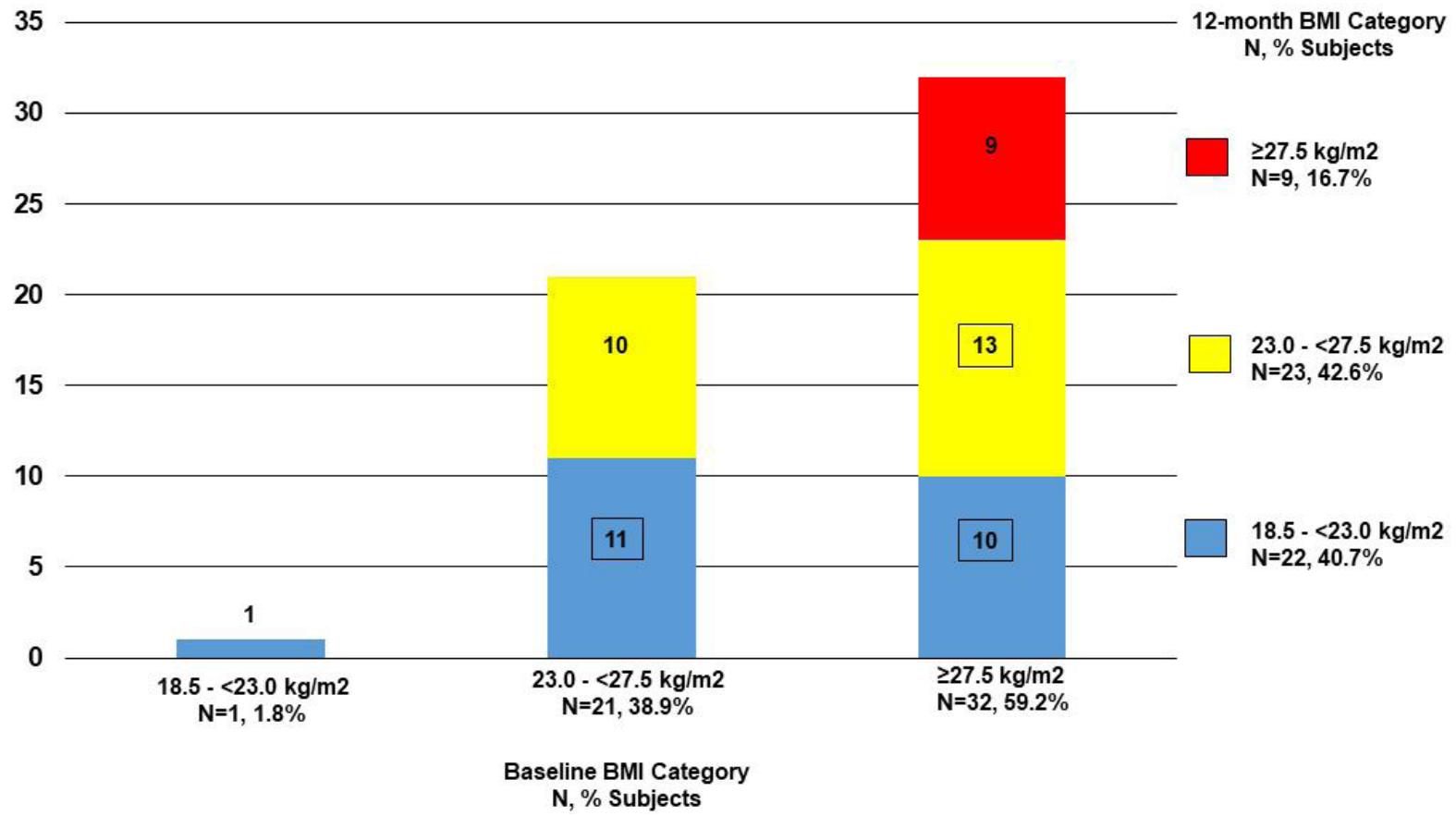

Figure 1. Change in BMI Risk Category after RYGB. The patients with T2DM ( $N=54)$ were placed into three groups, dependent on their baseline BMI. The $x$-axis shows the baseline BMI category distribution, while the $y$-axis shows the redistribution of the BMI groups at 12 months post-surgery. RYGB: Roux-en-Y gastric bypass; T2DM: type 2 diabetes mellitus; BMI: body mass index.

(19.7\%) achieved the defined remission criteria at 12 months after surgery. Preoperative factors in patients with and without T2DM remission were assessed [Table 4]. Univariable analyses identified the age and T2DM duration as being significantly lower and baseline BMI and weight as being significantly higher in the remission group than those in the non-remission group. These findings were confirmed in the multivariable analyses, although the small sample size of subjects with complete data at 12 months limited the generalizability of the results from the regression model. 


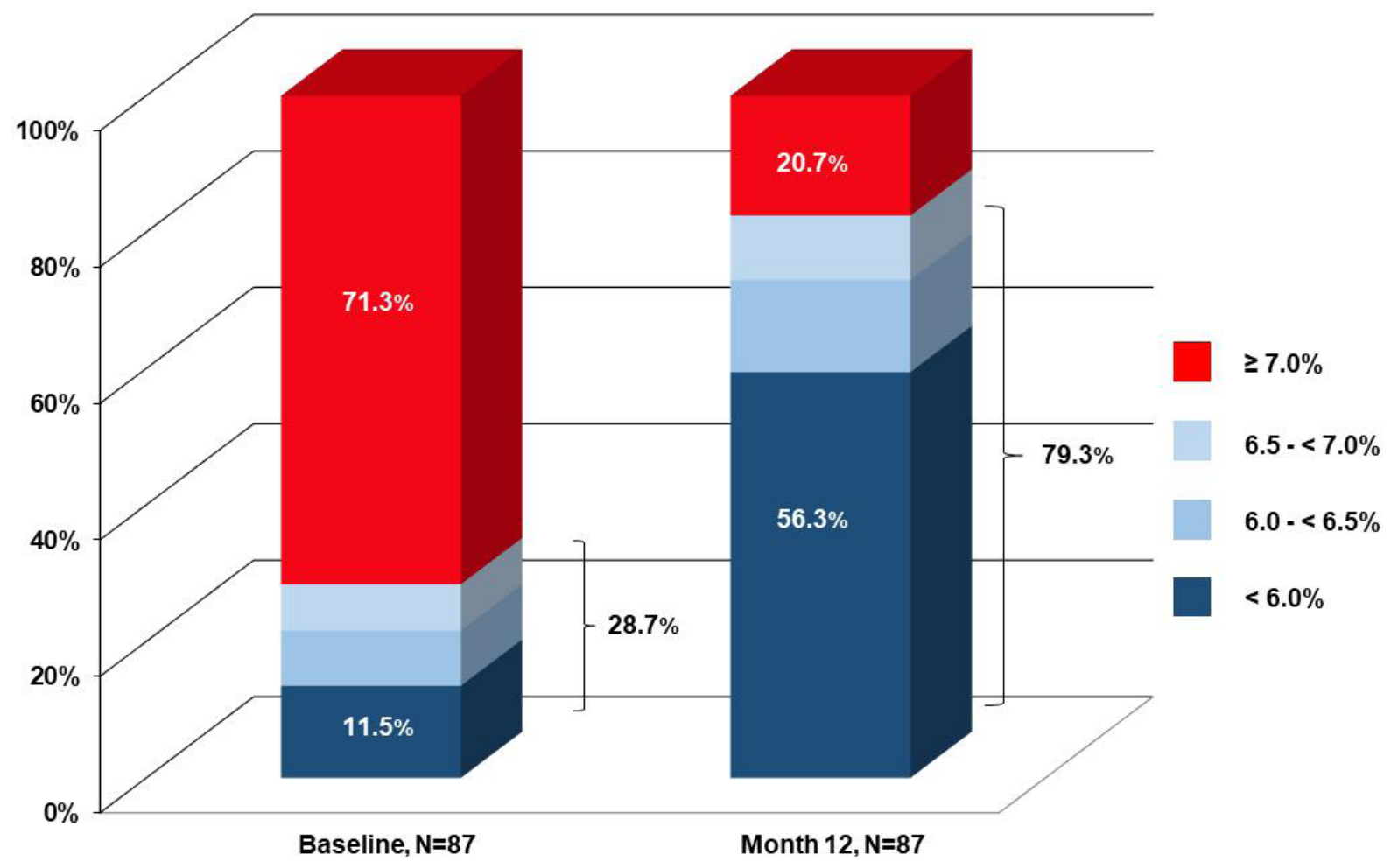

Figure 2. Glycemic control based on HbA1c for patients with T2DM. Patients were separated into four different HbA1c ranges $(N=87)$. The increase in the number of patients under glycemic control $(\mathrm{HbA1c}<6.0 \%)$ and the decrease in the number of patients with a high HbA1c ( $\geq 7.0 \%$ ) are shown. T2DM: Type 2 diabetes mellitus; HbA1c: glycosylated hemoglobin.

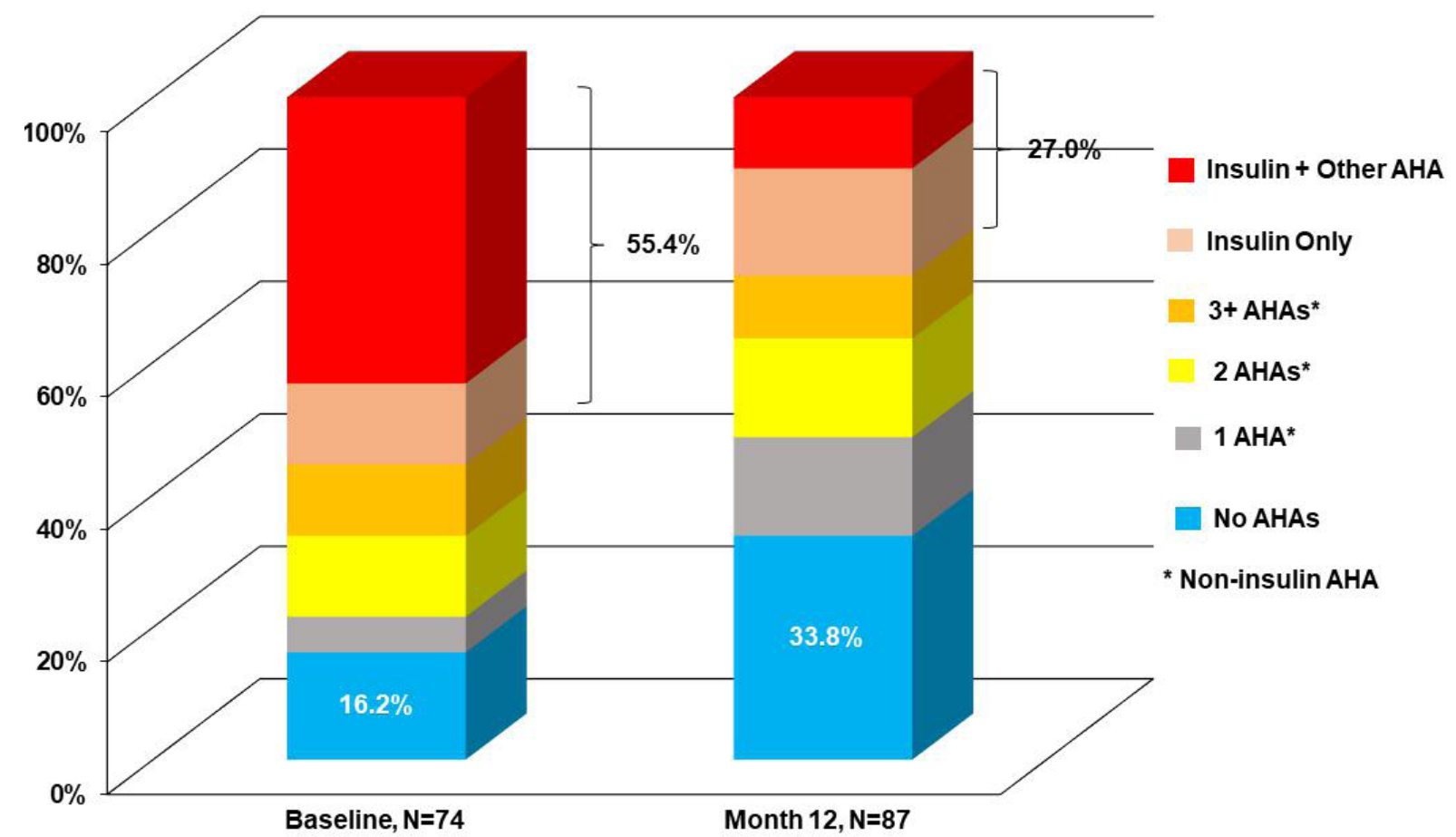

Figure 3. Medication use (anti-hyperglycemic agents) for patients with T2DM ( $N=74)$. The decrease in insulin usage is shown. T2DM: Type 2 diabetes mellitus. 
Table 4. Univariable analysis of preoperative and postoperative factors in patients with and without T2DM remission

\begin{tabular}{llll}
\hline Factor & $\begin{array}{c}\text { Remission } \\
(\boldsymbol{n}=\mathbf{1 4})\end{array}$ & $\begin{array}{c}\text { No remission } \\
(\boldsymbol{n}=\mathbf{5 7})\end{array}$ & $\boldsymbol{P}$ \\
\hline Age (years) & $37.8 \pm 8.4$ & $47.2 \pm 9.8$ & 0.002 \\
Weight (kg) & $109.3 \pm 36.1$ & $86.2 \pm 19.8$ & 0.035 \\
BMI (kg/m ${ }^{2}$ ) & $36.3 \pm 10.1$ & $30.5 \pm 6.9$ & 0.013 \\
Waist circumference (cm) & $115.6 \pm 25.7$ & $102.8 \pm 16.6$ & 0.184 \\
Fasting blood glucose (mg/dL) & $188.4 \pm 77.9$ & $163.8 \pm 63.5$ & 0.218 \\
HbA1c (\%) & $8.6 \pm 2.2$ & $7.8 \pm 1.9$ & 0.197 \\
Duration of T2DM (years) & $3.1 \pm 3.6$ & $6.2 \pm 4.9$ & 0.030 \\
Number of T2DM medications & $1.8 \pm 1.9$ & $2.4 \pm 1.4$ & 0.249 \\
Weight change (kg) & $-25.0 \pm 13.2$ & $-18.2 \pm 17.2$ & 0.295 \\
Percent weight change (\%) & $-26.4 \pm 10.5$ & $-18.6 \pm 13.1$ & 0.123 \\
BMl change $\left(\mathrm{kg} / \mathrm{m}^{2}\right)$ & $-8.4 \pm 4.6$ & $-6.6 \pm 6.3$ & 0.435 \\
\hline
\end{tabular}

Data are shown as mean \pm standard deviation. Results are consistent with the results of multivariable logistic regression, although the sample size was too small to support formal statistical modeling robustly. †Remission was defined as fasting blood glucose $<110 \mathrm{mg} / \mathrm{dL}$, $\mathrm{HbA1c}<6.0 \%$, and without the use of anti-hyperglycemic agents at 12 months after surgery. BMI: Body mass index; HbA1c: glycosylated hemoglobin; T2DM: type 2 diabetes mellitus.

\section{DISCUSSION}

This study showed that RYGB may be effective for weight loss and control of T2DM in Chinese patients who are obese and overweight considering the low remission rate. Patients with T2DM who were younger, had a higher BMI at baseline, and had a shorter T2DM duration were more likely to achieve T2DM remission. In patients with $\mathrm{T} 2 \mathrm{DM}$, significant improvements in anthropometric characteristics were observed at 12 months after surgery. Significant and meaningful improvements were concurrently observed in glycemic and lipid measurements. The outcomes reported in this retrospective study for RYGB appear consistent with recently published literature seen in Western patients with $\mathrm{T}_{2} \mathrm{DM}^{[11]}$ and Asian patients with $\mathrm{T} 2 \mathrm{DM}^{[15]}$.

Nevertheless, a major difference should be noted. In Western countries, about $80 \%$ of the patients who undergo bariatric surgery are female, mainly because of greater worries about the physical appearance and higher awareness of the impact of overweight on health than $\operatorname{men}^{[16]}$. In the present study, most patients were male (65\%). In the study of the bariatric surgeries performed between 2001 and 2015 in China, Du et al..$^{[5]}$ reported that males represented $48 \%$ of the patients, significantly more than in Western countries. The exact reason for this discrepancy is difficult to explain, as highlighted by Du et al. ${ }^{[5]}$, and additional study is necessary.

The WHO has previously presented health action points for BMI categories in Asian populations. The suggested categories were: underweight, $<18.5 \mathrm{~kg} / \mathrm{m}^{2}$; increasing but acceptable risk, $18.5-23 \mathrm{~kg} / \mathrm{m}^{2}$; increased risk, $23-27.5 \mathrm{~kg} / \mathrm{m}^{2}$; and high-risk, $\geq 27.5 \mathrm{~kg} / \mathrm{m}^{2[4]}$. The Diabetes Surgery Summit II (DSS-II) concluded that there is sufficient clinical and mechanistic evidence to support the inclusion of metabolic surgery among antidiabetic interventions for patients with T2DM and obesity, and it should be considered for Asian patients with T2DM and BMI $27.5-32.4 \mathrm{~kg} / \mathrm{m}^{2}$ if hyperglycemia is inadequately controlled with either oral or injectable medications ${ }^{[17]}$. In this study, we found that there was a redistribution of the BMI groups at 12 months after RYGB. In patients in the high-risk category $\left(\geq 27.5 \mathrm{~kg} / \mathrm{m}^{2}\right)$ at baseline, risk was reduced by one or more categories in $\geq 70 \%$ of patients, and, among those in the increased risk category $\left(23.0\right.$ to $\left.<27.5 \mathrm{~kg} / \mathrm{m}^{2}\right)$ at baseline, over $50 \%$ reached the increasing but acceptable risk category $(18.5$ to $<$ $\left.23.0 \mathrm{~kg} / \mathrm{m}^{2}\right)$. This result is consistent with the conclusions made by the DSS-II.

In the present study, the T2DM remission rate at 12 months was $19.7 \%$, which is lower than that reported in the Swedish Obese Subject study, where the remission rate with surgery was $72.3 \%$ at two years, but it decreased to $30.4 \%$ at 15 years ${ }^{[18]}$. A meta-analysis reported a remission rate of $78.1 \%^{[19]}$. The exact criteria 
for remission and the timing of evaluation may affect the results. In the present study, using strict criteria, remission was observed in $19.7 \%$, but near-remission was observed in the remaining $80.3 \%$ of the patients. Of note, LOCF had to be used to account for missing values in many patients.

Nevertheless, this study confirms the results of other work on the effectiveness of RYGB for weight loss in China and contributes to the growing body of evidence that RYGB can slow the progression of weightrelated diabetes, even inducing remission in some and improving control with fewer AHAs in the vast majority. Notably, more than half of those on insulin at baseline achieved glycemic control without insulin at 12 months after RYGB surgery. Preventing or reducing the need for insulin treatment is important both from a patient's quality of life perspective and from a healthcare utilization perspective ${ }^{[20]}$. In the present study, RYGB allowed at least a partial remission in all patients. Those with a short T2DM duration were at a higher likelihood of achieving remission, while those with a longer duration can nevertheless obtain some benefits from RYGB. Previous studies generally agree that younger age, shorter duration of diabetes, higher C-peptide levels, higher baseline BMI, and higher baseline visceral fat area are associated with remission after surgery ${ }^{[21-30]}$. Three prediction models based on different combinations of those variables are available (the DiaRem, ABCD, and individualized metabolic surgery scores) ${ }^{[21,31-33]}$. In the present study, no score could be derived from the data because of the limited data, but age and T2DM duration were lower and baseline BMI and weight were higher in the remission group than in the non-remission group, as supported by the previous models ${ }^{[21,31-33]}$ and studies ${ }^{[21-30]}$. Nevertheless, patients with higher BMI at baseline had a higher probability of achieving remission than those with a lower BMI. There is currently no accepted explanation for this phenomenon, but there is the possibility that the disease characteristics (such as insulin resistance and other metabolic disturbances) are different between the two groups of patients ${ }^{[21]}$. This will have to be examined using metabolic studies to determine possible differences in energy metabolism among patients that could account for the differences in weight loss. Because the BMI cutoff points are not the same between Chinese and Western patients, it is possible that the percent change in excess weight loss (\%EWL) is also different. In the present study, the $\% \mathrm{EWL}$ was $-42.8 \% \pm 44.2 \%$, indicating that, although the excess weight was cut by half in most patients, there was a wide variability among patients. In addition, \%EWL was not associated with remission, while some previous Western studies associated \%EWL with remission ${ }^{[11,23]}$. A meta-analysis showed ethnic differences in \%EWL after metabolic surgery, although Asian patients were not included ${ }^{[34]}$. In addition, around $60 \%$ of the patients in this study had a BMI lower than $32.5 \mathrm{~kg} / \mathrm{m}^{2}$, which may be very different from Western populations.

In the present study, the operative time and length of stay were longer than those usually observed in Western countries. The present study covered the 2009-2014 period, and Du et al ${ }^{[5]}$ showed that, even though bariatric surgery has been performed in China since 2001, most of the cases were in the 2011-2015 period, suggesting that the experience during 2009-2014 was relatively low, leading to longer surgeries. Regarding the length of stay, there is a shortage of general practitioners in China, and the Chinese healthcare system is based on specialists ${ }^{[35]}$. Therefore, patients are generally discharged when all symptoms and signs are resolved, leading to longer lengths of stay.

\section{STRENGTHS AND LIMITATIONS}

The strength of this report is the multicenter approach of data collection, capturing data from five Chinese hospitals. This report provides one of a limited number of multicenter studies available from China ${ }^{[12]}$. This study has several limitations, including the retrospective design, no comparative arm, a single procedure (RYGB) evaluated, exclusion of patients without 12-month data, a small patient population, lack of complete outcome data reported on the majority of patients at 12 months (e.g, BMI values were only available in 78 out of $103 \mathrm{~T} 2 \mathrm{DM}$ subjects), and the short-term follow-up. The data were from the first sites in China that conducted RYGB surgery, and it took time for patients to accept the new treatment pathway. 
Further work in prospective, multicenter, long-term follow-up designed studies is warranted to support RYGB as an effective, long-lasting treatment option in both morbidly and non-morbidly obese Chinese patients with T2DM.

In conclusion, This study supplements the evidence showing that RYGB is an important surgical option for the control of obesity and weight-related T2DM in Chinese patients. Half of the patients with insulin requirements at the time of RYGB can expect to maintain glycemic control with non-insulin AHAs after RYGB. Those not taking insulin prior to RYGB can expect to achieve glycemic control with fewer AHAs, and, if a patient has a short T2DM duration, glycemic control can even be achieved without the need for AHAs.

\section{DECLARATIONS}

\section{Acknowledgments}

Funding for this study has been provided by Ethicon Endo-Surgery, Inc.

\section{Authors' contributions}

Execution, data collection and interpretation, manuscript preparation, and final approval: Yang W Execution, data collection, and manuscript review and final approval: Zhu S, Cheng Z, Zhang N, Wu L, Chen Y, Yang J, Yu S

Clinical study design, data interpretation, and manuscript review and final approval: Yang TF, Ding D Clinical study design, data interpretation, manuscript preparation, and final approval: Waggoner JR Clinical study design, data interpretation, manuscript review and final approval: Schwiers ML, Fegelman EJ Execution, data collection and interpretation, and manuscript review and final approval: Wang CC

\section{Availability of data and materials}

Not applicable.

\section{Financial support and sponsorship}

Funding for this study has been provided by Ethicon Endo-Surgery, Inc.

\section{Conflicts of interest}

Yang W, Zhu S, Cheng Z, Zhang N, Wu L, Chen L, Yang J, Yu S, Wang CC declare that they have no conflict of interest. Yang TF and Ding D are employed by Johnson \& Johnson Medical (Shanghai) LTD. Waggoner JR, Schwiers ML and Fegelman EJ are employed by Ethicon Endo-Surgery, Inc.

\section{Ethical approval and consent to participate}

This study was approved by the Ethical Committee of the First Affiliated Hospital of Jinan University, Guangzhou, China. It also was registered on the Chinese Clinical Trial Registry website (www.chictr.org. cn) with Registration Number: ChiCTR-OOC-15006387.

\section{Consent for publication}

Not applicable.

\section{Copyright}

(c) The Author(s) 2021.

\section{REFERENCES}

1. World Health Organization [Internet]. Global report on diabetes; 2016. Available from:http://apps.who.int/iris/bitstre am/10665/204871/1/9789241565257_eng.pdf?ua=1\&ua=1 [Last accessed on 26 Feb 2021] 
2. Morton JM. Ethnic Considerations for Metabolic Surgery. Diabetes Care 2016;39:949-53.

3. International Diabetes Federation [Internet]. IDF diabetes atlas 8th edition; 2017 Available from: https://www.idf.org/e-library/ epidemiology-research/diabetes-atlas/134-idf-diabetes-atlas-8th-edition.html [Last accessed on 26 Feb 2021]

4. Appropriate body-mass index for Asian populations and its implications for policy and intervention strategies. The Lancet 2004;363:15763.

5. Du X, Dai R, Zhou HX, et al. Bariatric Surgery in China: How Is This New Concept Going? Obes Surg 2016;26:2906-12.

6. O'Neill S, O'Driscoll L. Metabolic syndrome: a closer look at the growing epidemic and its associated pathologies. Obes Rev 2015;16:112.

7. Ruan Y, Mo M, Joss-Moore L, et al. Increased waist circumference and prevalence of type 2 diabetes and hypertension in Chinese adults: two population-based cross-sectional surveys in Shanghai, China. BMJ Open 2013;3:e003408.

8. Gujral UP, Pradeepa R, Weber MB, Narayan KM, Mohan V. Type 2 diabetes in South Asians: similarities and differences with white Caucasian and other populations. Ann N Y Acad Sci 2013;1281:51-63.

9. Hou X, Lu J, Weng J, et al; China National Diabetes and Metabolic Disorders Study Group. Impact of waist circumference and body mass index on risk of cardiometabolic disorder and cardiovascular disease in Chinese adults: a national diabetes and metabolic disorders survey. PLoS One 2013;8:e57319.

10. Hales CM, Fryar CD, Carroll MD, Freedman DS, Ogden CL. Trends in Obesity and Severe Obesity Prevalence in US Youth and Adults by Sex and Age, 2007-2008 to 2015-2016. JAMA 2018;319:1723-5.

11. Schauer PR, Mingrone G, Ikramuddin S, Wolfe B. Clinical Outcomes of Metabolic Surgery: Efficacy of Glycemic Control, Weight Loss, and Remission of Diabetes. Diabetes Care 2016;39:902-11.

12. Wang G, Zhu L, Li W, Yang X, Li P, Zhu S. Can low BMI Chinese patients with type 2 diabetes benefit from laparoscopic Roux-en-Y gastric bypass surgery? Surg Obes Relat Dis 2016;12:1890-5.

13. Liang H, Guan W, Yang Y, et al. Roux-en-Y gastric bypass for Chinese type 2 diabetes mellitus patients with a BMI $<28 \mathrm{~kg} / \mathrm{m}(2)$ : a multi-institutional study. J Biomed Res 2015;29:112-7.

14. Agha R, Abdall-Razak A, Crossley E, Dowlut N, Iosifidis C, Mathew G; STROCSS Group. STROCSS 2019 Guideline: Strengthening the reporting of cohort studies in surgery. Int J Surg 2019;72:156-65.

15. Zhang P, Zhang H, Han X, et al. Effectiveness and safety of laparoscopic Roux-en-Y gastric bypass for the treatment of type 2 diabetes mellitus. Exp Ther Med 2016;11:827-31.

16. Fuchs HF, Broderick RC, Harnsberger CR, et al. Benefits of bariatric surgery do not reach obese men. J Laparoendosc Adv Surg Tech A 2015;25:196-201.

17. Rubino F, Nathan DM, Eckel RH, et al; Delegates of the 2nd Diabetes Surgery Summit. Metabolic Surgery in the Treatment Algorithm for Type 2 Diabetes: A Joint Statement by International Diabetes Organizations. Surg Obes Relat Dis 2016;12:1144-62.

18. Sjöström L, Peltonen M, Jacobson P, et al. Association of bariatric surgery with long-term remission of type 2 diabetes and with microvascular and macrovascular complications. JAMA 2014;311:2297-304.

19. Buchwald H, Estok R, Fahrbach K, et al. Weight and type 2 diabetes after bariatric surgery: systematic review and meta-analysis. Am $J$ Med 2009;122:248-256.e5.

20. Kalkan A, Bodegard J, Sundström J, et al. Increased healthcare utilization costs following initiation of insulin treatment in type 2 diabetes: A long-term follow-up in clinical practice. Prim Care Diabetes 2017;11:184-92.

21. Park JY. Prediction of Type 2 Diabetes Remission after Bariatric or Metabolic Surgery. J Obes Metab Syndr 2018;27:213-22.

22. Hayes MT, Hunt LA, Foo J, Tychinskaya Y, Stubbs RS. A model for predicting the resolution of type 2 diabetes in severely obese subjects following Roux-en Y gastric bypass surgery. Obes Surg 2011;21:910-6.

23. Hamza N, Abbas MH, Darwish A, Shafeek Z, New J, Ammori BJ. Predictors of remission of type 2 diabetes mellitus after laparoscopic gastric banding and bypass. Surg Obes Relat Dis 2011;7:691-6.

24. Blackstone R, Bunt JC, Cortés MC, Sugerman HJ. Type 2 diabetes after gastric bypass: remission in five models using HbA1c, fasting blood glucose, and medication status. Surg Obes Relat Dis 2012;8:548-55.

25. Aarts EO, Janssen J, Janssen IM, Berends FJ, Telting D, de Boer H. Preoperative fasting plasma C-peptide level may help to predict diabetes outcome after gastric bypass surgery. Obes Surg 2013;23:867-73.

26. Schauer PR, Bhatt DL, Kirwan JP, et al; STAMPEDE Investigators. Bariatric surgery versus intensive medical therapy for diabetes-3-year outcomes. N Engl J Med 2014;370:2002-13.

27. Bhasker AG, Remedios C, Batra P, Sood A, Shaikh S, Lakdawala M. Predictors of Remission of T2DM and Metabolic Effects after Laparoscopic Roux-en-y Gastric Bypass in Obese Indian Diabetics-a 5-Year Study. Obes Surg 2015;25:1191-7.

28. Yu H, Di J, Bao Y, et al. Visceral fat area as a new predictor of short-term diabetes remission after Roux-en-Y gastric bypass surgery in Chinese patients with a body mass index less than $35 \mathrm{~kg} / \mathrm{m}^{2}$. Surg Obes Relat Dis 2015;11:6-11.

29. Park JY, Kim YJ. Prediction of Diabetes Remission in Morbidly Obese Patients After Roux-en-Y Gastric Bypass. Obes Surg 2016;26:74956.

30. Scopinaro N, Adami GF, Bruzzi P, Cordera R. Prediction of Diabetes Remission at Long Term Following Biliopancreatic Diversion. Obes Surg 2017;27:1705-8

31. Still CD, Wood GC, Benotti P, et al. Preoperative prediction of type 2 diabetes remission after Roux-en-Y gastric bypass surgery: a retrospective cohort study. Lancet Diabetes Endocrinol 2014;2:38-45.

32. Lee WJ, Hur KY, Lakadawala M, et al. Predicting success of metabolic surgery: age, body mass index, C-peptide, and duration score. Surg Obes Relat Dis 2013;9:379-84. 
33. Aminian A, Brethauer SA, Andalib A, et al. Individualized Metabolic Surgery Score: Procedure Selection Based on Diabetes Severity. Ann Surg 2017;266:650-7.

34. Admiraal WM, Celik F, Gerdes VE, Dallal RM, Hoekstra JB, Holleman F. Ethnic differences in weight loss and diabetes remission after bariatric surgery: a meta-analysis. Diabetes Care 2012;35:1951-8.

35. Andrews B, Bullock MB. Medical transitions in twentieth-century China. Bloomington \& Indianapolis: Indiana University Press; 2014. 